\title{
Temperatura como um fator de risco para internações hospitalares em 70 cidades do MS
}

\author{
Temperature as a risk factor for hospital admissions in 70 cities MS
}

\author{
Amaury de Souza e Debora A. da Silva Santos \\ Universidade Federal de Mato Grosso do Sul, Instituto de Física. MS ,Brasil \\ amaury.de@uol.com.br \\ Universidade Federal de Mato Grosso, Depto de Enfermagem, MG, Brasil \\ deboraassan@hotmail.com
}

\begin{abstract}
Resumo
O objetivo deste estudo foi quantificar a carga de morbidade total de internação por doenças respiratórias atribuível a temperatura ambiente não-ideal e as contribuições relativas de calor e frio e de temperaturas moderadas e extremas. Foram coletados dados diários destas internações de 70 locais de cidades de Mato Grosso do Sul e de temperaturas máxima e mínimas do ar. Para verificar esta relação utilizou-se o modelo aditivo generalizado de regressão de quasi Poisson. A análise foi ajustada para sazonalidades de longa e curta duração, temperaturas mínima e máxima, sendo adotado nível de significância $\alpha=5 \%$. Calculou-se internações atribuíveis para calor e frio, definidas como temperaturas acima e abaixo da temperatura óptima e para temperaturas moderadas e extremos, definidos usando ponto de corte o 2, 5 the 97, 5 th • percentis. Foram analisados 148.849 internações em vários períodos entre 2004 e 2009. No total, 6,62\% (95\% de IC- 6.53-6.82) deveu-se a temperatura não óptima nas cidades selecionadas. O percentil temperatura de morbidade mínima variou de aproximadamente o $60^{\circ}$ percentil. As temperaturas atribuíveis para internação foram causadas pelo frio $(6,38 \%$, 95\% de IC 6,04-6,58)do que pelo calor $(0,39 \%, 0,28$ 0,42). Temperaturas quentes e frias extremas foram responsáveis por $(0,75 \% ; 0,71-0,79 \%)$. da morbidade total.
\end{abstract}

Palavras-chave: Temperatura alta; Temperatura baixa; Hospitalização.

\begin{abstract}
The aim of this study was to quantify the overall disease burden of hospitalization for respiratory diseases attributable to non-ideal room temperature and the relative contributions of heat and cold, moderate and extreme temperatures. Were collected daily data from these admissions of 70 local cities of Mato Grosso do Sul and the maximum and minimum temperatures. To verify this relationship we used the generalized additive model quasi Poisson regression. The analysis was adjusted for seasonality long and short duration, minimum and maximum temperatures being adopted significance level $\alpha=5 \%$. hospitalization was calculated attributable to heat and cold, defined as temperatures above and below the optimum temperature and moderate and extreme temperatures using defined cutoff point 2, 5 and 97 th, 5 th percentile $\bullet$. 148,849 hospitalizations were analyzed at various times between 2004 and 2009. In total, 6.62\% (95\% IC-6.53-6.82) was due to non-optimal temperature in selected cities. The percentile temperature minimal morbidity ranged from approximately the 60th percentile. Temperatures attributable to hospitalization were caused by cold $(6.38 \%, 95 \%$ CI 6.04 to 6.58$)$ than heat $(0.39 \%, 0.28$ to 0.42$)$. extreme hot and cold temperatures accounted for $(0.75 \%, 0.71-0.79 \%)$. of the total morbidity.
\end{abstract}

Keywords: High Temperature; Low temperature; Hospitalization. 


\section{Introdução}

Muitos estudos epidemiológicos têm fornecido evidências para a associação entre a temperatura ambiente e de resultados mortalidade /morbidade (BASU; SAMET; 2002; YE et al., 2012). Interesse neste tópico aumentou após episódios de clima extremo e em resposta aos relatórios sobre as mudanças climáticas (LE TERTRE et al., 2006; SEMENZA et al., 1996; IPCC, 2013).

Embora exista consenso entre os pesquisadores que ambas as temperaturas extremamente frias e muito quentes afetam a saúde, a sua importância relativa é uma questão de debate atual e outros detalhes da associação continuam inexplorados. Por exemplo, pouco se sabe sobre as temperaturas ideais que correspondem aos efeitos mínimos para vários resultados de saúde. Além disso, a maioria dos estudos tem-se concentrado sobre os eventos extremos e não há estudos que têm avaliado relativamente à contribuição de temperaturas moderadamente elevadas e baixas. Os mecanismos fisiopatológicos subjacentes que ligam a exposição à temperatura não ideal e risco de morbidade não foram completamente elucidados. A insolação em dias quentes e hipotermia em dias frios representam apenas pequenas proporções de internações/mortes em excesso. Temperaturas altas e baixas foram associadas com risco aumentado para uma ampla gama de doenças cardiovasculares, respiratórias, e de outras causas, sugerindo a existência de várias vias biológicas (ANALITIS et al., 2008; ANDERSON; BELL, 2009; GASPARRINI et al., 2012; THE EUROWINTER GROUP, 1997).

Temperatura ambiente representa um fator de risco importante e mais estudos são necessários para reforçar a compreensão dos efeitos de saúde associados. Esta informação é essencial para o planejamento das intervenções de saúde pública adequada e para o fornecimento de previsões confiáveis para os efeitos das alterações climáticas.

Estudos epidemiológicos sobre o tema enfrentam desafios importantes na modelação de dependências da temperatura com a saúde. Em primeiro lugar, a relação dose-resposta, o que é inerentemente não linear, também é caracterizado por diferentes períodos de atraso para o calor e o frio, isto é, o excesso de risco causado pelo calor é tipicamente imediato e ocorre dentro de alguns dias, enquanto que os efeitos do frio a relato que duraram até 3 ou 4 semanas (ANALITIS et al., 2008; ANDERSON; BELL, 2009). Em segundo lugar, a associação é heterogênea entre as populações por causa de aclimatação, diferentes respostas de adaptação e variabilidade nos fatores de susceptibilidade (ZANOBETTI et al., 2013; HAJAT; O'CONNOR; KOSATSKY,2010; McMICHAEL et al., 2008).

Embora os estudos tenham quantificado a associação, em termos de risco relativo (RR), poucos deram estimativas de carga imputável, quer como excesso absoluto (números) ou excesso relativo (frações) de internações
(BACCINI et al., 2011; HAJAT; ARMSTRONG; BACCINI, et al. 2006; CARSON et al., 2006; HUYNEN et al., 2001; ROONEY et al., 1998; PENG et al., 2011; BASAGANA et al., 2011). A evidencia para o risco atribuível da temperatura é muitas vezes restrita a eventos extremos (ROONEY et al., 1998; PENG et al., 2011), especialmente as ondas de calor, embora algumas investigações têm relatado valores das associações de dose-resposta estimados em modelos com a temperatura como uma variável contínua (BACCINI et al., 2011; HAJAT et al., 2006).

Uma nova concepção de saúde teve origem em meados da década de 70, no Canadá, com Lalonde e Laframboise, que trabalharam a concepção do processo saúde-doença dentro de uma nova perspectiva, considerando o amplo contexto que envolve esse processo. Criaram, então, o conceito de "Campo de Saúde", que passou a ser um modelo adotado pelo governo canadense. Tal modelo preconiza que a saúde é determinada por um conjunto de 4 fatores de risco: biologia humana, estilo de vida, ambiente e sistemas de organização de cuidados à população [LESSA, 1994].

Assim, ao se considerar que saúde tem como determinante, não apenas as condições biológicas, mas também as ambientais e as relativas ao estilo de vida, os fatores como qualidade do ar, do solo e da água passam a ter uma importância relevante, pois, ao se alterar as condições naturais desses sistemas básicos da vida, podemos ter disparado um processo de desequilíbrio na saúde de grupos ou indivíduos expostos a essa nova situação, da mesma forma como ocorre com os fatores socioeconômicos, culturais e políticos.

O objetivo deste estudo foi quantificar o número de internações hospitalar total atribuível à temperatura ambiente não ideal, e as contribuições relativas de calor e frio e de temperaturas moderadas e extremas. Baseamos a nossa análise sobre os recentes avanços em modelagem estatística para explicar a complexa e heterogênea dependência da temperatura-morbidade.

A seleção de Mato Grosso do Sul para este estudo se deve a sua localização geográfica. Têm como limites os Estados de Goiás a nordeste, Minas Gerais a leste, Mato Grosso (norte), Paraná (sul) e São Paulo (sudeste), além da Bolívia (oeste) e o Paraguai (oeste e sul). Possui uma área de 358.124,962 km2, com uma população de aproximadamente 2.360 .498 habitantes, segundo as estimativas populacionais do IBGE para o ano de 2010, com 79 municípios e predominância em atividades primárias, sua economia está baseada na produção rural (animal, vegetal, extrativa vegetal e indústria rural), indústria, extração mineral, turismo e prestação de serviços. Mato Grosso do Sul possui um dos maiores rebanhos bovinos do país.

\section{Metodologia}

“O Estado do Mato Grosso do Sul está localizado 
entre as latitudes 17o13 '40" e 19o27'47" S; e longitudes de 50o56 '06" e 53o42'18" W.

Trata-se de um estudo do tipo ecológico de série espacial e temporal, em que foi analisada a tendência do coeficiente de doença respiratória, no Estado de Mato Grosso do Sul.

Os dados de internações hospitalares por causas respiratórias (Capítulo X - Doenças do aparelho respiratório que compreende as categorias de J00 até J99 da Classificação Internacional de Doenças - CID 10) segundo local de residência para cada município do Estado de Mato Grosso do Sul foram obtidos nos bancos de dados informatizados do Ministério da Saúde, através das Autorizações de Internações Hospitalares (AIH) do Sistema Único de Saúde (SUS) para o ano de 2005-2009 (DATASUS, 2012).

Laurenti et al. (1985) definiu o coeficiente de incidência hospitalar como:

\section{$\mathrm{C}=\mathrm{I} / \mathrm{POP}^{*} 1000$}

I representa o número de internações hospitalares em um município, e pop a população deste município.

As doenças respiratórias usadas na análise para o estado de Mato Grosso do Sul, extraídas do Sistema de Informações Hospitalares (SIH) do SUS (DATASUS), foram analisadas no período de estudo de 1827 dias (01 de janeiro de 2005 a 31 de dezembro de 2009), o número de internações por doenças respiratórias foram de 148.849 .

Em Mato Grosso do Sul, os dados relativos ao crescimento e desenvolvimento econômico e social encontram no: Índice de Desenvolvimento Humano Municipal - IDH-M, importantes indicadores sobre o desenvolvimento econômico e também social das cidades, por diferentes regiões, propiciando um perfil de grande relevância para as políticas públicas.

A interrelação desses indicadores revela resultados incisivos sobre a qualidade de vida da população. Por isso, em avaliação de situação de saúde, este é um importante indicador a ser considerado, se tomarmos como referência o conceito sistêmico do processo saúde-doença, ou seja, o entendimento da interconexão de todos os fatores envolvidos e que determinam os diferentes estágios da vida humana. Foi realizado um estudo de correlações entre internações hospitalares, temperaturas mínimas, máximas e o IDH-M para cada município. O Índice de Desenvolvimento Humano Municipal (IDH-M) é o índice que mede o desenvolvimento humano no nível municipal. As dimensões avaliadas são renda, educação e longevidade.

Neste estudo optou-se pela temperatura média diária como o índice de exposição, calculado a partir de estações de monitorização centrais, tanto como a média entre os 24 h, média valores máximos e mínimos. Foi realizada uma análise de sensibilidade, modificando as opções de modelagem, substituindo todas as causas com a morbidade não externo e controle de poluição do ar/focos de queimadas e umidade no subgrupo das cidades.

O presente estudo fundamenta-se em dados secundários, de acesso público, que não constrangerão grupos de populações e/ou indivíduos na apresentação dos resultados encontrados, sendo assegurada a confidencialidade das informações levantadas. Sendo assim, foram respeitados os aspectos éticos de pesquisa com seres humanos, de acordo com a Resolução $\mathrm{n}^{\mathrm{o}}$ 466/2012 (BRASIL, 2012).

\section{Análise estatística}

Toda a análise foi feita com a utilização do software $\mathrm{R}$, utilizando os pacotes dlnm e mvmeta. O código está disponível sob pedido, e um exemplo de reproduzível está incluído no site pessoal do autor Antônio Gasparini. Foi aplicado pela primeira vez uma série temporal regressão padrão quasi-Poisson separadamente em cada local para obter estimativas de associações de temperatura de morbidade específica por localização, classificado como RR [BHASKARAN et al., 2013].

Resumidamente, essa regressão de primeira fase incluiu uma B-spline cúbico natural do tempo com 8 graus de liberdade por ano para controlar as tendências sazonais e de longo prazo, e um indicador de dia da semana. Nós modelamos a associação com a temperatura utilizando um atraso modelo de distribuídos não linear [GASPARRINI, 2014].

Esta classe de modelos pode descrever complexa relação não linear e dependências desfasada, através da combinação de duas funções que definem a associação de exposição-resposta convencional e a associação resposta lag- adicional, respectivamente. A associação lag-resposta representa a variação temporal do risco após uma exposição específica, e estima a distribuição de efeitos imediatos e retardados que acumulam ao longo do período lag. Especificamente, nós modelamos a curva de exposição-resposta com uma B-spline quadrática com três nós internos colocados no 10th, 75th, e 90th percentis das distribuições de temperatura específicas em localização, e a curva de lag-resposta com uma B-spline cúbico natural com uma intercepção e três nós internos colocados em valores igualmente espaçados na escala log.

Nós estendemos o período lag 21 dias para incluir o atraso dos efeitos do frio e para excluir as internações que foram incluídas por apenas alguns dias. Testamos essas escolhas de modelos em análise de sensibilidade. Nós então reduzimos à associação da temperatura de morbidade, acumulando o risco durante o período de atraso [GASPARRINI; ARMSTRONG, 2013]. Este passo reduz o número de parâmetros a serem agrupados na análise de segunda fase, e preserva a complexidade da dependência estimada, evitando-se assim a simplificação desnecessária. 
Estudos relataram como fatores econômicos, demográficos e de infraestrutura climatológicos, social, ter um papel na modificação da associação entre temperatura e morbidade. Para ter em conta as principais características de tal modificação de efeito, incluímos a temperatura de localização específica média, faixa de temperatura, e os indicadores para as cidades como preditores em uma regressão multivariada. Testamos esses efeitos através de um teste de Wald multivariada. Testamos heterogeneidade residual e relatou que pela extensão multivariada de teste de Cochran Q [ZANOBETTI et al., 2013; GASPARRINI, ARMSTRONG, KENWARD, 2012; HIGGINS; THOMPSON, 2002].

Utilizou-se o modelo analítico para derivar a melhor predição linear da associação de exposição-resposta cumulativa total em cada local. A melhor predição linear representa uma compensação entre a associação de localização específica fornecida pela regressão de primeira fase e a associação agrupada. Esta abordagem permite que as áreas com contagens pequenas de morbidade diária ou série curta, geralmente caracterizados por estimativas muito imprecisas, para pedir informações a partir de populações maiores que compartilham características semelhantes [BACCINI, et al., 2011; GASPARRINI, ARMSTRONG, KENWARD, 2012; POST et al., 2001].

A temperatura mínima da morbidade, o que corresponde a um percentil mínimas de morbidade entre o primeiro e os percentis 99 th, foi derivada a partir da melhor predição linear da associação de exposição-resposta cumulativa total em cada local. Nós nos referimos a este valor como a temperatura ideal, e considerou a referência para o cálculo do risco atribuível por re-centrar a B-spline quadrática que modela a exposição-resposta. Para cada dia da série, em cada local, utilizou-se a RR cumulativo correspondente a temperatura de cada dia para calcular as internações atribuíveis e fração de internação atribuíveis nos próximos 21 dias, utilizando um método previamente descrito [GASPARRINI; LEONE, 2014].

O número total de internações causadas por temperaturas não óptimas é dada pela soma das contribuições de todos os dias da série, e sua relação com o número total de internações fornece a fração atribuível total. Foram calculados os componentes atribuíveis ao frio e ao calor pela soma dos subconjuntos correspondentes de dias com temperaturas superiores ou inferiores do que a temperatura mínima da morbidade. Separamos ainda mais essas componentes em contribuições moderadas e extremas através da definição de frio e calor extremos, como temperaturas mais baixas do que a 2,5 th percentil, localização específica (frio extremo) e maior do que o 97,5 th percentil do local específico (calor extremo). Estes cortes são consistentes com as definições anteriores de condições meteorológicas extremas, tais como ondas de calor. Definimos temperaturas moderadas, como as gamas entre a temperatura ideal e esses cortes. Definimos outras faixas usando pontos de corte para os 10 th, 25 th, 50 th, 75 th, e 90th percentis.

A análise de agrupamento tem como objetivo identificar grupos homogêneos dentro de uma população (CORRAR et al., 2007; WILKS, 2006). Neste trabalho é usado o método de classificação hierárquico, com critério de agrupamento proposto por Ward (1963). A distância utilizada para medir a similaridade ou dissimilaridade dos dados foi à euclidiana padronizada ao quadrado.

\section{Resultados}

O conjunto de dados incluiu 148.849 internações hospitalares. Como esperado, as populações em diferentes cidades experimentaram uma ampla gama de temperaturas, com médias específicas de cada cidade variam com uma média de $15,7^{\circ} \mathrm{C}$ e uma média mínima de $13,9{ }^{\circ} \mathrm{C}$ com um valor máximo mínimo de $19,4{ }^{\circ} \mathrm{C}$, para os valores máximos a média foi: $33,3^{\circ} \mathrm{C}$ com um uma média mínima de $30,5{ }^{\circ} \mathrm{C}$ e uma média máxima de $36,8 \stackrel{\circ}{\circ}$ conforme figura 1 .

O IDH-M teve um valor médio de 0.68 com um mínimo 0.59 para a cidade de Tacuru e Coronel Sapucaí e um valor máximo de 0.78 para a cidade de Campo Grande.

A tabela 1 mostra a média da curva de exposição cumulativa global para 70 cidades selecionadas, com a temperatura a morbidade mínima correspondente e os pontos de corte para definir temperaturas extremas. As distribuições de temperatura enfatizam como a gama de temperatura de ar quente, embora caracterizado por um elevado RR, consiste em apenas uma grande percentagem de dias. As faixas de morbidade percentuais mínimos médios foram de cerca de percentil 60, resultados também coincidentes com os de Gasparini et al., (2015) para o Brasil. Risco aumenta lentamente e linearmente para temperaturas frias abaixo da temperatura mínima de morbidade, embora alguns locais mostraram um aumento maior para o frio extremo do que os outros. Em contraste, o risco geralmente aumentado rapidamente e de forma não linear em altas temperaturas.

As parcelas em 3-D mostram as relações não lineares entre a temperatura média e internações hospitalares específicas por idade por doenças respiratórias ao longo de 21 dias de atraso (Figura 2). Para o total de admissões hospitalares, não houve efeito evidente da temperatura, mas ambas as temperaturas baixas e altas foram associadas com aumento do número de admissão total hospitalar. Observou-se que as temperaturas altas e baixas foram associadas com um aumento do número de internações respiratórias em pessoas com mais de 60 anos, enquanto os riscos relativos de admissões mais elevados a temperaturas frias em outros grupos etários. 

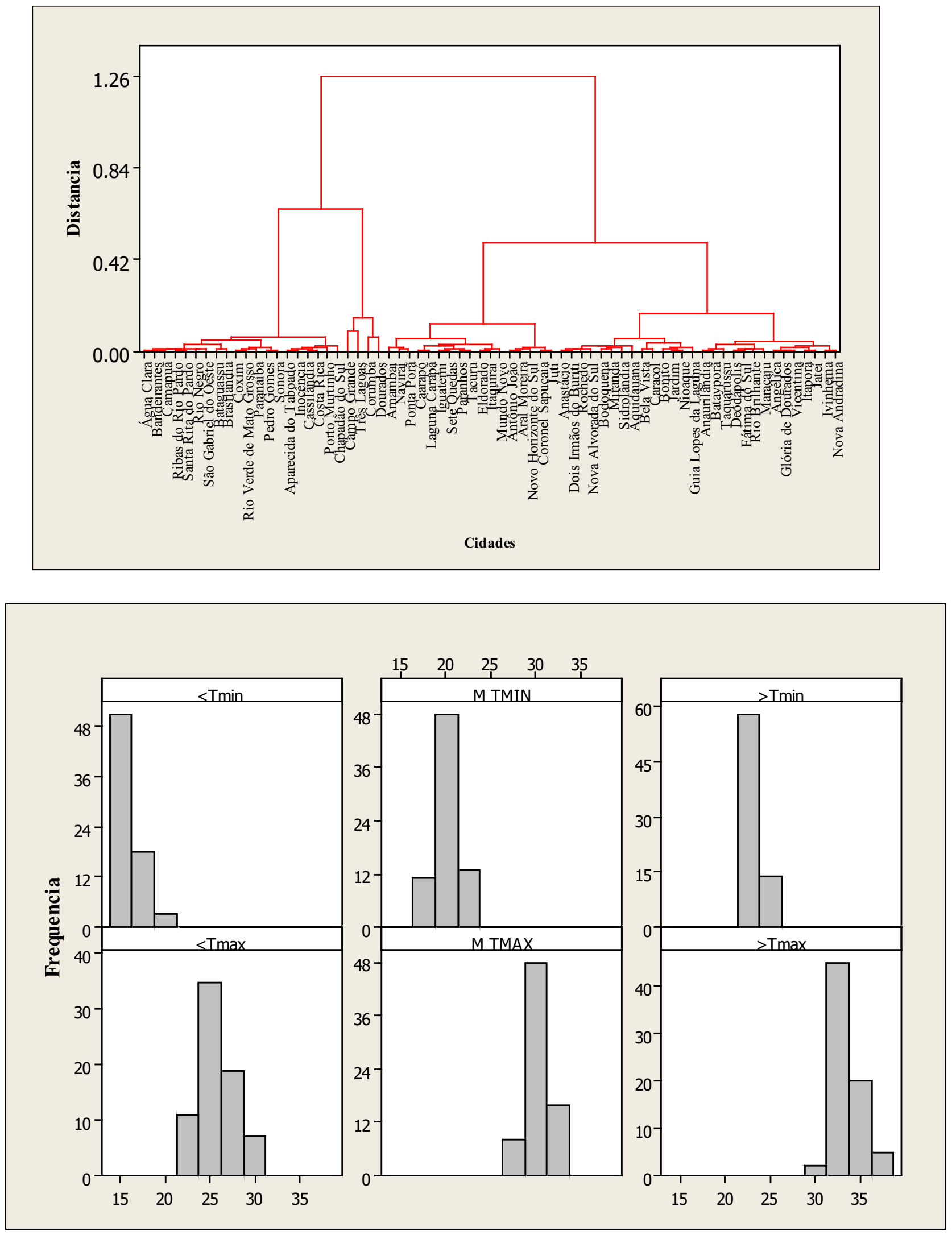

Figura 1 - Histograma das distribuições das medias das temperaturas mínima e máxima das cidades de MS. 


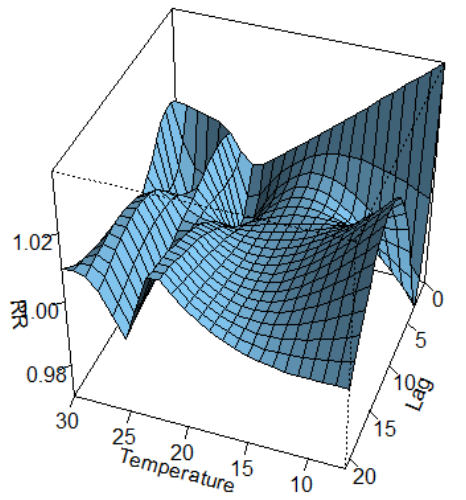

All

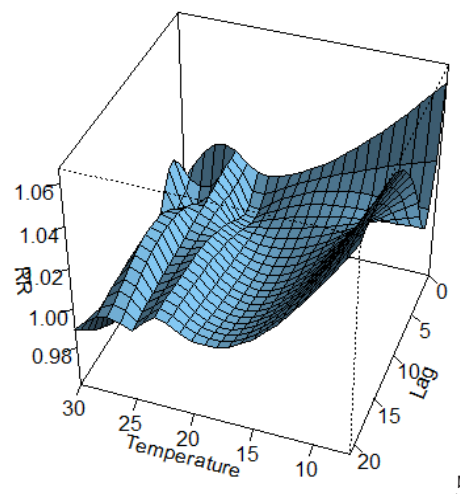

$5-60$

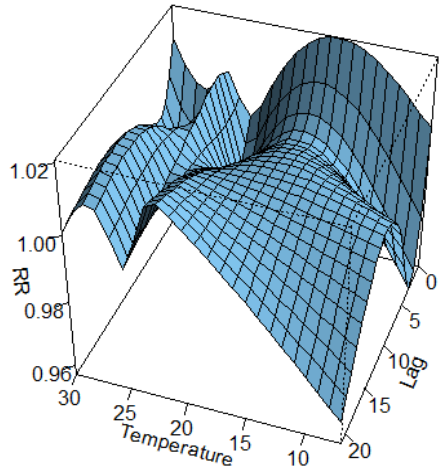

$0-4$

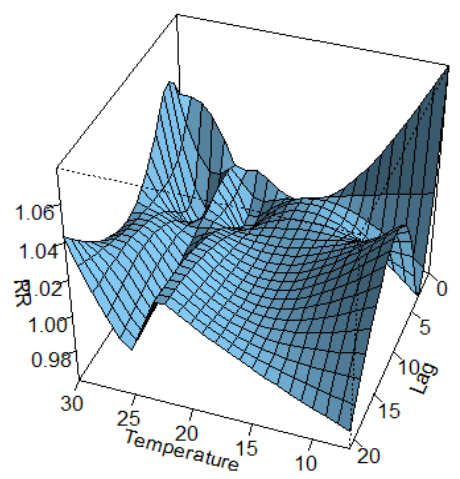

$>60$

Figura 2 - Gráfico tridimensional da associação entre a temperatura média e internações hospitalares específicas por idade ao longo de 21 dias.

Os resultados da regressão multivariada sugerem que, apesar de significativa, a heterogeneidade residual é baixa após indicadores das cidades, a temperatura média e o IDH haviam sido incluídos como preditores. Embora todos os preditores modificam significativamente a associação de morbidade com temperatura, seja um preditor simples ou modelos completos, os indicadores das cidades são responsáveis por uma proporção muito maior de heterogeneidade do que a temperatura média ou faixa de temperatura.

Os principais resultados (tabela 1) foram à fração atribuível estimado calculado como totais e como componentes separados causadas por temperaturas quentes e frias em cada cidade. No geral, a fração total de internações causadas pela exposição ao calor e frio foi de 6,62\% (95\% de IC- 6.53-6.82). Frio foi responsável pela maior parte da carga (total estimativa $(6,38 \%, 95 \%$ de IC 6,04-6,58), enquanto a fração atribuível ao calor foi pequena $(0,39 \%, 0,28-0,42)$. Esta diferença foi causada principalmente pelas altas percentil-morbidade mínima, com a maioria das temperaturas médias diárias sendo menor do que o valor óptimo.

O risco atribuível pode ser separado em componentes relacionados a temperaturas moderadas e extremas (tabela 1). Em todos as cidades, a maior parte do risco de morbidade atribuível a temperatura foi relacionado ao frio moderado, com uma estimativa global de $(5,46 \%$ e IC- 5,31-5,78). As temperaturas extremas (frio ou quente) foram responsáveis por uma fração pequena, correspondendo a $(0,75 \%$ 0,71-0,79\%). Estes resultados são consistentes com as associações de exposição-resposta e distribuições de temperatura. Embora o correspondente gama de moderada a frio tinha uma RR comparativamente baixo, que inclui a maioria dos dias na série. Nossa análise de sensibilidade sugeriu que nossos resultados não foram dependentes das premissas de modelagem. 
Tabela 1 - Grupo e subgrupos das cidades de MS, analisados pelo agrupamento de Wards, medias das temperaturas mínimas $\left({ }^{\circ} \mathrm{C}\right)$, medias das temperaturas máximas $\left({ }^{\circ} \mathrm{C}\right)$ e RR-risco relativo para as temperaturas mínimas e máximas calculados pelas regressão de quasi Poisson.

\begin{tabular}{|c|c|c|c|c|c|}
\hline Municípios & $\begin{array}{l}\text { sub } \\
\text { grupo }\end{array}$ & Media TMIN & Media TMAX & RRTmin & RRTmax \\
\hline Água Clara & 1.1 & $20,42(23,30-15,90)$ & $30,90(34,40-26,40)$ & $1,1611(1,21911,1030)$ & $1,6170(1,6978-1,5361)$ \\
\hline Amambai & 2.1 & $18,59(21,90-14,10)$ & $28,70(31,90-23,70)$ & $1,3177(1,3835-1,2518)$ & $1,5553(1,6330-1,4775)$ \\
\hline Anastácio & 2.1 & $21,38(24,00-17,10)$ & $32,93(36,10-28,60)$ & $1,6611(1,7442-1,5780)$ & $1,0612(1,1142-1,0081)$ \\
\hline Anaurilândia & 2.2 & $19,81(22,80-15,50)$ & $30,30(33,60-25,60)$ & $1,4250(1,4962-1,3537)$ & $1,8319(1,9235-1,7403)$ \\
\hline Angélica & 2.2 & $19,58(22,60-15,20)$ & $30,02(32,80-25,20)$ & $1,5786(1,6576-1,4997)$ & $1,9431(2,0403-1,8460)$ \\
\hline Antônio João & 2.1 & $19,43(22,20-15,50)$ & $29,53(32,00-25,00)$ & $1,6616(1,7446-1,5785)$ & $1,1486(1,2060-1,0912)$ \\
\hline $\begin{array}{l}\text { Aparecida do } \\
\text { Taboado }\end{array}$ & 1.1 & $20,64(23,40-16,60)$ & $30,73(34,70-26,50)$ & 1,1317(1,1883-1,0752) & $1,6769(1,7608-1,5931)$ \\
\hline Aquidauana & 2.1 & $21,38(24,00-17,10)$ & $32,93(36,10-28,60)$ & $1,6611(1,7442-1,5780)$ & $1,0612(1,1142-1,0081)$ \\
\hline Aral Moreira & 2.1 & $18,71(21,70-14,50)$ & $28,53(31,50-23,70)$ & $1,3177(1,3835-1,2518)$ & $1,6454(1,7277-1,5631)$ \\
\hline Bandeirantes & 1.1 & $20,12(22,50-16,50)$ & $30,28(33,40-26,70)$ & $1,0753(1,1290-1,0215)$ & $1,8383(1,9302-1,7464)$ \\
\hline Bataguassu & 1.1 & $20,03(22,90-15,80)$ & $30,56(34,00-25,90)$ & $1,3196(1,3856-1,2537)$ & $1,7361(1,8229-1,6493)$ \\
\hline Batayporã & 2.2 & $19,36(22,60-15,00)$ & $29,79(32,60-24,90)$ & $1,7046(1,7899-1,6194)$ & $1,0362(1,0880-0,9844)$ \\
\hline Bela Vista & 2.1 & $20,45(23,50-16,30)$ & $31,29(34,10-26,40)$ & $1,1611(1,2191-1,1030)$ & $1,4905(1,5650-1,4159)$ \\
\hline Bodoquena & 2.1 & $20,53(23,40-16,00)$ & $31,88(35,10-27,50)$ & $1,8761(1,9699-1,7823)$ & $1,3179(1,3838-1,2520)$ \\
\hline Bonito & 2.1 & $19,619(22,60-15,30)$ & $30,68(33,60-26,20)$ & $1,2221(1,2832-1,1610)$ & $1,6915(1,7761-1,6069)$ \\
\hline Brasilândia & 1.1 & $20,42(23,10-16,40)$ & $30,71(34,30-26,00)$ & $1,2863(1,3506-1,2220)$ & $1,6827(1,7669-1,5986)$ \\
\hline Caarapó & 2.1 & $18,73(21,80-14,10)$ & $29,12(32,20-24,40)$ & $1,9374(2,0343-1,8405)$ & $1,3431(1,4103-1,2760)$ \\
\hline Camapuã & 1.1 & $20,02(22,40-16,20)$ & $30,36(33,90-26,80)$ & $1,0481(1,1005-0,9957)$ & $1,8098(1,9003-1,7193)$ \\
\hline Campo Grande & 1.2 & $19,89(22,00-16,20)$ & $29,80(32,70-26,10)$ & $1,2538(1,3165-1,1911)$ & $1,0327(1,0843-0,9810)$ \\
\hline Caracol & 2.1 & $20,48(23,90-16,20)$ & $31,11(34,00-26,00)$ & $1,2863(1,3506-1,2220)$ & $1,5484(1,6258-1,4710)$ \\
\hline Cassilândia & 1.2 & $19,30(22,00-15,10)$ & $29,59(33,50-25,90)$ & $1,3196(1,3856-1,2537)$ & $1,1227(1,1788-1,0666)$ \\
\hline Chapadão do Sul & 1,1 & $19,48(21,90-15,90)$ & $29,52(33,10-26,20)$ & $1,2221(1,2832-1,1610)$ & $1,1561(1,2139-1,0983)$ \\
\hline Coronel Sapucaia & 2.1 & $18,97(22,40-14,60)$ & $28,98(32,30-23,80)$ & $2,2591(2,3720-2,1461)$ & $1,4090(1,4795-1,3386)$ \\
\hline Corumbá & 1.2 & $22,34(25,20-17,70)$ & $33,55(36,80-29,00)$ & $1,5968(1,6766-1,5169)$ & $1,9318(2,0284-1,8352)$ \\
\hline Costa Rica & 1.1 & $19,51(22,10-16,20)$ & $28,99(32,80-26,30)$ & $1,1912(1,2508-1,1316)$ & $1,4049(1,4751-1,3346)$ \\
\hline Coxim & 1.1 & $22,40(25,00-18,60)$ & $32,77(36,20-29,50)$ & $1,5251(1,5513-1,4988)$ & $1,0967(1,1515-1,0418)$ \\
\hline Deodápolis & 2.2 & $19,13(22,40-14,70)$ & $29,58(32,50-24,70)$ & $1,7942(1,8839-1,7045)$ & $1,1264(1,1827-1,0701)$ \\
\hline Dois Irmãos do Buriti & 2.1 & $21,46(23,80-17,50)$ & $32,36(35,50-28,30)$ & $1,7139(1,7996-1,6282)$ & $1,1939(1,2536-1,1342)$ \\
\hline Dourados & 1.2 & $19,26(22,10-14,50)$ & $29,97(32,80-25,30)$ & $1,5387(1,6157-1,4618)$ & $1,9634(2,0616-1,8653)$ \\
\hline Eldorado & 2.1 & $18,63(22,40-14,00)$ & $28,60(32,20-22,90)$ & $1,8444(1,9366-1,7522)$ & $1,6090(1,6894-1,5285)$ \\
\hline Fátima do Sul & 2.2 & $19,06(22,20-14,40)$ & $29,57(32,60-24,70)$ & $1,7942(1,8839-1,7045)$ & $1,1338(1,1905-1,0771)$ \\
\hline Glória de Dourados & 2.2 & $19,13(22,40-14,70)$ & $29,58(32,50-24,70)$ & $1,7942(1,8839-1,7045)$ & $1,1264(1,1827-1,0701)$ \\
\hline Guia Lopes da Laguna & 2.1 & $20,20(22,90-16,20)$ & $30,98(33,60-26,50)$ & $1,1317(1,1883-1,0752)$ & $1,5892(1,6686-1,5097)$ \\
\hline Iguatemi & 2.1 & $18,66(22,40-14,10)$ & $28,89(32,30-23,50)$ & $1,4394(1,5114-1,3674)$ & $1,4554(1,5282-1,3826)$ \\
\hline Inocência & 1.1 & $19,97(22,40-16,20)$ & $29,91(33,60-26,00)$ & $1,2863(1,3506-1,2220)$ & $1,9874(2,0868-1,8880)$ \\
\hline Itaporã & 2.2 & $19,26(22,10-14,50)$ & $29,97(32,80-25,30)$ & $1,5387(1,6157-1,4618)$ & $1,9634(2,0616-1,8653)$ \\
\hline Itaquiraí & 2.1 & $18,82(22,60-14,30)$ & $29,08(32,50-23,70)$ & $1,3177(1,3835-1,2518)$ & $1,3635(1,4317-1,2954)$ \\
\hline Ivinhema & 2.2 & $19,13(22,40-14,70)$ & $29,58(32,50-24,70)$ & $1,7942(1,8839-1,7045)$ & $1,1264(1,1827-1,0701)$ \\
\hline Jardim & 2.1 & $20,20(22,90-16,20)$ & $30,98(33,60-26,50)$ & $1,1317(1,1883-1,0752)$ & $1,5892(1,6686-1,5097)$ \\
\hline Jateí & 2.2 & $19,06(22,20-14,40)$ & $29,57(32,60-24,70)$ & $1,7942(1,8839-1,7045)$ & $1,1338(1,1905-1,0771)$ \\
\hline Juti & 2.1 & $18,84(22,30-14,30)$ & $29,29(32,60-24,10)$ & $1,0921(1,1467-1,0375)$ & $1,2594(1,3223-1,1964)$ \\
\hline Laguna Carapã & 2.1 & $18,73(21,80-14,10)$ & $29,12(32,20-24,40)$ & $1,9374(2,0343-1,8405)$ & $1,3431(1,4103-1,2760)$ \\
\hline Maracaju & 2.2 & $19,87(22,40-15,70)$ & $30,28(32,80-25,90)$ & $1,3196(1,3856-1,2537)$ & $1,8415(1,9335-1,7494)$ \\
\hline Miranda & 2.1 & $21,94(24,70-17,40)$ & $33,35(36,70-28,80)$ & $1,6281(1,7095-1,5467)$ & $1,9714(2,0699-1,8728)$ \\
\hline
\end{tabular}


Tabela 1 - Continuação...

\begin{tabular}{|c|c|c|c|c|c|}
\hline Municípios & $\begin{array}{l}\text { sub } \\
\text { grupo }\end{array}$ & Media TMIN & Media TMAX & RRTmin & RRTmax \\
\hline Mundo Novo & 2.1 & $18,63(22,40-14,00)$ & $28,60(32,20-22,90)$ & $1,8444(1,9366-1,7522)$ & $1,6090(1,6894-1,5285)$ \\
\hline Naviraí & 2.1 & $18,98(22,50-14,50)$ & $29,41(32,60-24,20)$ & $1,0392(1,0912-0,9872)$ & $1,2052(1,2655-1,1449)$ \\
\hline Nioaque & 2.1 & $20,82(23,50-16,70)$ & $32,42(35,30-28,00)$ & $1,7709(1,8594-1,6823)$ & $1,1795(1,2385-1,1205)$ \\
\hline Nova Alvorada do Sul & 2.1 & $20,07(22,70-15,80)$ & $30,54(33,30-26,00)$ & $1,2863(1,3506-1,2220)$ & $1,7421(1,8292-1,6550)$ \\
\hline Nova Andradina & 2.2 & $19,56(22,70-15,20)$ & $29,97(32,80-25,20)$ & $1,5786(1,6576-1,4997)$ & $1,9634(2,0616-1,8653)$ \\
\hline $\begin{array}{l}\text { Novo Horizonte do } \\
\text { Sul }\end{array}$ & 2.1 & $19,13(22,40-14,70)$ & $29,58(32,50-24,70)$ & $1,7942(1,8839-1,7045)$ & $1,1264(1,1827-1,0701)$ \\
\hline Paranaíba & 1.1 & $20,11(23,10-15,90)$ & $30,33(34,30-26,30)$ & $1,1912(1,2508-1,1316)$ & $1,8192(1,9102-1,7283)$ \\
\hline Paranhos & 2.1 & $19,14(22,80-14,60)$ & $29,50(33,40-23,70)$ & $1,3177(1,3835-1,2518)$ & $1,1636(1,2217-1,1054)$ \\
\hline Pedro Gomes & 1.1 & $21,63(24,50-18,30)$ & $32,17(35,80-29,20)$ & $1,5670(1,6453-1,4886)$ & $1,2425(1,3046-1,1803)$ \\
\hline Ponta Porã & 2.1 & $18,48(21,40-14,30)$ & $28,21(30,90-23,70)$ & $1,3177(1,3835-1,2518)$ & $1,8304(1,9219-1,7389)$ \\
\hline Porto Murtinho & 1.1 & $22,77(25,30-19,20)$ & $33,21(36,40-30,00)$ & $1,4620(1,5351-1,3889)$ & $1,9123(2,0079-1,8167)$ \\
\hline Ribas do Rio Pardo & 1.1 & $19,53(22,40-15,20)$ & $29,67(32,70-25,50)$ & $1,4619(1,5350-1,3888)$ & $1,0898(1,1443-1,0354)$ \\
\hline Rio Brilhante & 2.2 & $19,72(22,60-15,10)$ & $30,43(33,40-25,60)$ & $1,4250(1,4962-1,3537)$ & $1,7849(1,8741-1,6956)$ \\
\hline Rio Negro & 1.1 & $21,53(24,10-18,00)$ & $31,98(35,20-28,50)$ & $1,6782(1,7622-1,5943)$ & $1,2907(1,3553-1,2262)$ \\
\hline $\begin{array}{l}\text { Rio Verde de Mato } \\
\text { Grosso }\end{array}$ & 1.1 & $21,86(24,50-18,20)$ & $32,02(35,40-28,60)$ & $1,6611(1,7442-1,5780)$ & $1,2818(1,3459-1,2177)$ \\
\hline Rochedo & 2.1 & $21,51(24,00-18,00)$ & $32,05(35,20-28,30)$ & $1,7139(1,7996-1,6282)$ & $1,2730(1,3366-1,2093)$ \\
\hline Santa Rita do Pardo & 1.1 & $20,05(23,00-15,70)$ & $30,35(33,70-25,70)$ & $1,3890(1,4584-1,3195)$ & $1,8129(1,9036-1,7223)$ \\
\hline São Gabriel do Oeste & 1.1 & $20,26(22,90-16,70)$ & $30,59(33,90-27,20)$ & $1,9461(2,0434-1,8488)$ & $1,7241(1,8103-1,6379)$ \\
\hline Sete Quedas & 2.1 & $18,48(22,20-13,90)$ & $28,46(32,10-22,80)$ & $1,9182(2,0141-1,8223)$ & $1,6870(1,7713-1,6026)$ \\
\hline Sidrolândia & 2.1 & $20,49(22,70-16,70)$ & $30,77(33,50-26,80)$ & $1,0481(1,1005-0,9957)$ & $1,6624(1,7456-1,5793)$ \\
\hline Sonora & 1.1 & $22,56(25,40-19,40)$ & $33,05(36,60-30,30)$ & $1,4278(1,4992-1,3564)$ & $1,0339(1,0856-0,9822)$ \\
\hline Tacuru & 2.1 & $18,57(22,20-14,10)$ & $28,54(31,90-23,30)$ & $1,5676(1,6459-1,4892)$ & $1,6408(1,7229-1,5588)$ \\
\hline Taquarussu & 2.2 & $19,36(22,60-15,00)$ & $29,79(32,60-24,90)$ & $1,7046(1,7899-1,6194)$ & $1,0362(1,0880-0,9844)$ \\
\hline Três Lagoas & 1.2 & $20,71(23,30-16,80)$ & $30,77(34,50-26,20)$ & $1,2221(1,2832-1,1610)$ & $1,6624(1,7456-1,5793)$ \\
\hline Vicentina & 2.2 & $19,06(22,20-14,40)$ & $29,57(32,60-24,70)$ & $1,7942(1,8839-1,7045)$ & $1,1338(1,1905-1,0771)$ \\
\hline
\end{tabular}

\section{Discussão}

Os resultados desta pesquisa mostram que a temperatura é responsável pelo avanço da fração substancial de internações, correspondendo a $6,62 \%$ da morbidade nas cidades selecionados dentro do período de estudo. A maior parte desta carga de morbidade foi causada por dias mais frios do que a temperatura ideal (6,38\%), em comparação com os dias mais quentes do que a temperatura ideal (0,39\%). Além disso, a maioria das internações foram causadas por exposição a temperaturas moderadamente quentes e frias, e a contribuição de dias extremos, foi comparativamente baixo, apesar do aumento RR. A análise dos dados de 70 locais fornece evidência para o risco de morbidade relacionada com a temperatura em uma ampla variedade de climas e populações com demográfica diferente, sócia econômica, e as características de infraestrutura. No estudo foram aplicados modelos estatísticos novos e flexíveis para caracterizar as estimativas de associação morbidade com temperatura em todos os locais. Em particular, embora estudos anteriores contassem com a simplificação da estrutura de exposição-resposta ou lag, a abordagem que usamos aqui nos permitiu estimar e dependências não lineares e retardados e identificar a temperatura da morbidade mínima.

A comparação com estudos anteriores que relataram dados de internações atribuíveis é limitada por vários fatores, principalmente a variação de desenhos de estudo e abordagens de modelagem e o uso de definições alternativas de medidas de risco atribuíveis. As constatações do estudo que incidiu sobre eventos ou períodos com temperaturas extremas específicas sugerem um aumento da morbidade de $8 \bullet 9-12 \bullet 1 \%$ durante as ondas de calor e $12 \bullet 8 \%$ durante período de frio [HUYNEN et al., 2001; ROONEY et al., 1998] que estendeu a análise para todo o verão, e a morbidade é atribuível causada pelo calor [BACCINI et al., 2011; BASAGANA et al., 2011] que incluem medidas de risco atribuíveis para a morbidade de todo o ano, e, portanto, adotar um denominador comparável: Hajat et al. (2006) informou que a todas as causas de morbidade /mortalidade atribuível ao calor era entre $0 \bullet 37 \%$ e $1 \bullet 45 \%$ em três cidades europeias, e Carson et al. (2006) estimaram que $5 \bullet 4 \%$ das mortes foram atribuídas ao frio mas nenhum para aquecer em Londres. 
Vários mecanismos subjacentes têm sido usados para explicar o aumento do risco de morbidade associado à exposição à temperatura ambiente alta e baixa. Efeitos fisiológicos que levam as internações/mortes relacionadas com o calor que ainda não estão bem conhecidas, e provavelmente variam para diferentes causas de morbidade/ mortalidade. No caso da associação de calor com a morbidade/mortalidade cardiovascular, a causa responsável pelo maior índice, eventos agudos parecem ser acionado quando o corpo excede o limite de termorregulação, depois de alterações do ritmo cardíaco, a viscosidade do sangue e coagulação, redução da perfusão cerebral, e atenuado vasoconstritor expansividade [KEATINGE et al.,1986]. O calor também aumenta o risco de morbidade/mortalidade por outras causas: um mecanismo sugerido é através da alteração do equilíbrio de fluidos e eletrolíticos em pessoas que sofrem de doenças crónicas ou em pessoas com capacidade de resposta diminuída às condições ambientais. [BASU; SAMET, 2002; GASPARRINI et al., 2012].

Sobre as estações na região metropolitana de São Paulo, Gonçalves e Coelho (2010) concluíram que, nos meses de abril e maio, existe uma tendência do aumento da temperatura do ar, consequentemente, da morbidade das doenças respiratórias, mais comumente em crianças e idosos, atribuída a coeficientes de correlações positivas, comparando as afecções de vias aéreas superiores e a temperatura mínima mensal.

Estas respostas fisiológicas são consistentes como aumento acentuado, do risco acima da temperatura óptima, o que foi associado com uma carga relativamente alta atribuível a uma temperatura extremamente alta. Os processos biológicos que sustentam a morbidade relacionada com o frio têm, principalmente, efeitos cardiovasculares e respiratórios. Exposição ao frio tem sido associada com o estresse cardiovascular afetando fatores como a pressão arterial e fibrinogênio plasma, vasoconstrição e viscosidade do sangue, e as respostas inflamatórias [KEATINGE et al., 1984; WOODHOUSE et al., 1994].

Da mesma forma, o frio induz broncoconstrição e suprime as defesas muco ciliar e outras reações imunológicas, resultando em inflamação local e aumento do risco de infecções respiratórias. Estas respostas fisiológicas podem persistir por mais tempo do que os atribuídos ao calor, e parecem produzir riscos de morbidade que seguem uma resposta suave, com a maior parte do risco atribuível ocorrendo em dias moderadamente frios. Algumas limitações devem ser reconhecidas. Primeiro, embora esta investigação inclua populações com características marcadamente diferentes e estar em uma grande variedade de climas, os resultados não podem ser interpretados como representativos globalmente. Nós não incluímos regiões inteiras, e a avaliação foi restrita principalmente às populações urbanas.

Embora nossos resultados sugiram variação substancial entre cidade em risco atribuível tanto para calor e frio, a análise não caracteriza estas diferenças para identificar determinantes de suscetibilidade ou resistência aos efeitos da temperatura. Essas limitações podem ser abordadas em pesquisas futuras, por extensão, do conjunto de dados para as populações que vivem em outras regiões, e pela coleção de medidas padronizadas com variáveis com as características específicas em localização para ser incluído em uma segunda regressão.

A temperatura ideal em que o risco é menor estava bem acima da média, e parecia ser aumentada em regiões frias. Frio foi responsável por uma proporção mais elevada de internações do que o calor, enquanto a moderadas temperaturas quentes e frias representou a maioria da carga total em saúde. A investigação sobre a associação entre a saúde humana e a temperatura ambiente, até agora focado principalmente sobre os efeitos do calor extremo, e os planos de saúde, têm implementado políticas e intervenções destinadas quase exclusivamente por períodos de calor. Nossos resultados sugerem que as políticas de saúde pública e medidas de adaptação devem ser alargadas e reorientadas para ter em conta toda a gama de efeitos associados com a temperatura, porém, mais pesquisas são necessárias para esclarecer o quanto o excesso de morbidade relacionada com cada componente é evitável. Nosso estudo também fornece uma plataforma para melhorar e alargar as previsões dos efeitos das alterações climáticas; nossos resultados enfatizam como uma avaliação completa é necessária para fornecer uma estimativa adequada das consequências para a saúde de vários cenários de mudanças climáticas.

A maioria dos municípios do Mato Grosso do Sul apresentaram altos valores de casos das doenças respiratórias, na região leste do estado, área que coincide com a presença de indústrias de extração de carvão, a relação direta entre população e casos de doenças respiratórias indica a vulnerabilidade das pessoas, uma vez que as indústrias se concentram na zona rural. Na região Norte do estado, o modelo apresenta os mesmos fatores, mas provavelmente a origem da emissão de monóxido de carbono não é derivada da região carbonífera, mas da queima da biomassa.

A variável população infantil (um a quatro anos de idade) apresentaram relação direta com as doenças respiratórias. Esse fato aponta a relação entre as condições climáticas e o agravo das doenças, as quais, nesse caso, são maiores nos períodos de estiagem. A seleção das variáveis no modelo gerado justifica-se por melhor explicar a ocorrência dos casos de doenças respiratórias. Variáveis ambientais e socioeconômicas que não se mantiveram no modelo, mas que tiveram forte associação com as variáveis selecionadas, também pode ser utilizado como fatores de risco para ocorrência de novos casos de doenças. Dentre as variáveis socioeconômicas, 'vínculos empregatícios na indústria de transformação', 'população urbana' e 'população de 65 anos e mais' mostraram-se correlacionadas com a população de 1 a 4 anos', presente no modelo, a qual permaneceu no modelo final. 
Também se deve considerar o tipo da vegetação, que é denominado como combustível, que neste caso a vegetação é do tipo: cerrado a Leste, Pantanal a Oeste, floresta tropical a Sul, e apresenta propriedades favoráveis a incêndios. As propriedades dos combustíveis envolvem o tipo, diâmetro, peso e umidade, dentre as quais o teor de umidade é a mais importante no controle de incêndio.

O conhecimento antecipado das condições meteorológicas pode ajudar a sociedade a evitar a degradação da natureza assim como prevenir doenças do aparelho respiratório (SOUZA, et al.; 2015).

\section{Conclusões}

Os resultados destas multi-cidades fornecem provas de uma atenuação do impacto da morbidade associada com o calor na maioria das cidades incluídos na análise. Estes resultados têm implicações importantes para a investigação das alterações climáticas. No cenário atual, a aplicação de dependências de morbidade temperatura determinado utilizando dados históricos podem sobrestimar o impacto de alta temperatura no futuro. Embora o uso da relação exposição-resposta previsto para o ano mais recente é provável que representem uma estimativa mais adequada, pelo menos para projeções de curto prazo, o quadro metodológico aqui proposta não fornece ferramentas para prever como a associação vai ainda mais mudar no futuro. A pesquisa futura deve identificar os condutores de mudanças temporais, fatores e mecanismos que modificam a suscetibilidade de uma população para os efeitos na saúde de calor. Além de fornecer a base para projeções mais rigorosas do impacto das alterações climáticas, esta prova será crucial para a melhoria das intervenções de saúde pública destinadas a reduzir as consequências para a saúde.

\section{Referências}

Analitis A, Katsouyanni K, Biggeri A, et al. Effects of cold weather on mortality: results from 15 European cities within the PHEWE Project. Am J Epidemiol. 2008;168:1397.

Anderson BG, Bell ML. Weather-related mortality: how heat, cold, and heat waves affect mortality in the United States. Epidemiology. 2009;20:205-13.

Baccini M, Kosatsky T, Analitis A, et al. Impact of heat on mortality in 15 European cities: attributable deaths under different weather scenarios. J Epidemiol Community Health. 2011;65:64-70.
Basagana X, Sartini C, Barrera-Gomez J, et al. Heat waves and cause-specific mortality at all ages. Epidemiology. 2011;22:765-72.

Basu R, Samet JM. Relation between elevated ambient temperature and mortality: a review of the epidemiologic evidence. Epidemiol Rev. 2002;24:190-202.

Bhaskaran K, Gasparrini A, Hajat S, Smeeth L, Armstrong B. Time series regression studies in environmental epidemiology. Int J Epidemiol. 2013;42:1187-95.

Brasil. Portaria no 466/2012 de outubro de 2012. Dispõe sobre diretrizes e normas regulamentadoras de pesquisa com seres humanos. Brasília (DF): Conselho Nacional de Saúde; 2012. Publicada no Diário Oficial da União de 13 de junho de 2013, Seção 1, p.59.

Carson C, Hajat S, Armstrong B, Wilkinson P. Declining vulnerability to temperature-related mortality in London over the 20th century. Am J Epidemiol. 2006. Jul 1;164(1):77-84.

Corrar LJ, Paulo E, Filho JMD. Análise Multivariada: para os cursos de administração, ciências contábeis e economia. Ed. Atlas. São Paulo, 2007.

DATASUS. Informações em Saúde. Disponível em http:// w3.datasus.gov.br/datasus/ datasus.php.

DATASUS. Sistemas e Aplicativos. Disponível em http:// www.datasus.gov.br/cid10/.

Gasparrini A, Armstrong B, Kenward MG. Multivariate meta-analysis for non-linear and other multi-parameter associations. Stat Med. 2012;31:3821-39.

Gasparrini A, Armstrong B, Kovats S, Wilkinson P. The effect of high temperatures on cause-specific mortality in England and Wales. Occup Environ Med. 2012;69:56-61.

Gasparrini A, Armstrong B. Reducing and meta-analyzing estimates from distributed lag non-linear models. BMC Med Res Methodo. 2013;13: 1.

Gasparrini A, Leone M. Attributable risk from distributed lag models. BMC Med Res Methodol. 2014;14:55.

Gasparrini A. Modeling exposure-lag-response associations with distributed lag non-linear models. Stat Med. 2014;33:881-99.

Gonçalves FLT, Coelho MSZS. Variação da morbidade de doenças respiratórias em função da variação da temperatura entre os meses de abril e maio em São Paulo. Ciência e Natura. 2010;32(1):103-118. 
Hajat S, Armstrong B, Baccini M, et al. Impact of high temperatures on mortality: is there an added heat wave effect? Epidemiology. 2006;17:632-38.

Hajat S, O'Connor M, Kosatsky T. Health effects of hot weather: from awareness of risk factors to effective health protection. Lancet. 2010;375:856-63.

Higgins JPT, Thompson SG. Quantifying heterogeneity in a meta-analysis. Stat Med. 2002;21:1539-58.

Huynen MM, Martens P, Schram D, Weijenberg MP, Kunst AE. The impact of heat waves and cold spells on mortality rates in the Dutch population. Environ Health Perspect. 2001;109:463-70.

IPCC. Climate change 2013: the physical science basis. Working group I contribution to the fifth assessment report of the intergovernmental panel on climate change. Cambridge, UK and New York, USA: Cambridge University Press, 2013.

Keatinge WR, Coleshaw SR, Cotter F, Mattock M, Murphy $\mathrm{M}$, Chelliah R. Increases in platelet and red cell counts, blood viscosity, and arterial pressure during mild surface cooling: factors in mortality from coronary and cerebral thrombosis in winter. BMJ. 1984;289:1405-08.

Keatinge WR, Coleshaw SR, Easton JC, Cotter F, Mattock $\mathrm{MB}, \mathrm{Chelliah} \mathrm{R}$. Increased platelet and red cell counts, blood viscosity, and plasma cholesterol levels during heat stress, and mortality from coronary and cerebral thrombosis. Am J Med. 1986;81:795-800.

Laurenti R, et al. Estatísticas de saúde. São Paulo: EPU/ Edusp;1985.

Lessa I. Doenças não transmissíveis.In: Rouquayrol, M.Z. (Ed.), Epidemiologia \& saúde. 4.ed. Rio de Janeiro: Medsi, 1994,269-279.

Le Tertre A, Lefranc A, Eilstein D, et al. Impact of the 2003 heatwave on all-cause mortality in 9 French cities. Epidemiology. 2006;17:75-79.

McMichael AJ, Wilkinson P, Kovats RS, et al. International study of temperature, heat and urban mortality: the 'ISOTHURM' project. Int J Epidemiol. 2008;37:1121-31.

Peng RD, Bobb JF, Tebaldi C, McDaniel L, Bell ML, Dominici F. Toward a quantitative estimate of future heat wave mortality under global climate change. Environ Health Perspect. 2011;119:701-06.

Post E, Hoaglin D, Deck L, Larntz K. An empirical Bayes approach to estimating the relation of mortality to exposure to particulate matter. Risk Anal. 2001;21:837-42.
Rooney C, McMichael AJ, Kovats RS, Coleman MP. Excess mortality in England and Wales, and in Greater London, during the 1995 heatwave. J Epidemiol Community Health. 1998;52:482-86.

Semenza JC, Rubin CH, Falter KH, et al. Heat-related deaths during the July 1995 heat wave in Chicago. N Engl J Med. 1996;335:84-90.

Souza A, Aristone F, Pavao HG, Kofanovski AZ, Santos DAS. Impacto do ambiente atmosférico nas internações hospitalares por doenças respiratórias. Espacios. 2015;36(24):12.

The Eurowinter Group. Cold exposure and winter mortality from ischaemic heart disease, cerebrovascular disease, respiratory disease, and all causes in warm and cold regions of Europe. Lancet. 1997;349:1341-46.

Ward, JH. Hierarchical grouping to optimize an objective function. Journal of the American Statistical Association. 1963;58(301):236-244.

Wilks, DS. Statistical Methods in the Atmospheric Sciences. 2a Edition. California: Elsevier Science \& Technology Books. Academic Press, 2006.

Woodhouse PR, Khaw KT, Plummer M, Foley A, Meade TW. Seasonal variations of plasma fibrinogen and factor VII activity in the elderly: winter infections and death from cardiovascular disease. Lancet. 1994;343:435-39.

Ye X, Wolff R, Yu W, Vaneckova P, Pan X, Tong S. Ambient temperature and morbidity: a review of epidemiological evidence. Environ Health Perspect. 2012;120:19-28.

Zanobetti A, O'Neill MS, Gronlund CJ, Schwartz JD. Susceptibility to mortality in weather extremes: effect modification by personal and small-area characteristics. Epidemiology. 2013;24:809-19. 